\title{
Adding fear to conflict: A general purpose cognitive control network is modulated by trait anxiety
}

\author{
Marie K. Krug and Cameron S. Carter \\ University of California, Davis, California
}

\begin{abstract}
Studies of cognitive control show that the dorsal anterior cingulate cortex (dACC) and dorsolateral prefrontal cortex are involved in the detection and resolution of cognitive conflict. However, the neural and behavioral mechanisms underlying emotional interference effects are less consistent. We used fMRI during emotional and nonemotional versions of a facial Stroop task to investigate the effects of emotional stimuli on cognitive control. In the full group there was limited evidence that different prefrontal circuits manage conflict arising from emotional and nonemotional distractors. However, individual differences in trait anxiety affected both behavioral performance and neural activity during the emotional task. Relative to low-anxiety (LA) subjects, high-anxiety (HA) subjects showed greater amygdala activity to task-relevant emotional information and impaired performance and greater conflict-related activity in the dACC when emotional content was task-irrelevant. Only LA subjects activated rostral ACC during the emotional task. This is consistent with cognitive models of individual differences that hypothesize deficient control of task-irrelevant emotional information in HA subjects. Additional behavioral and fMRI results from this study may be downloaded from http://cabn.psychonomic-journals .org/content/supplemental.
\end{abstract}

Cognitive control forms a foundation for higher cognitive functions such as attention, memory retrieval, and language production and comprehension. Cognitive control allows us to coordinate or direct lower level or more automatic processes to ensure that our resulting actions will be in line with our goals and to successfully perform difficult tasks, even in the face of distraction. While the behavioral and neural mechanisms of cognitive control are well characterized, a growing body of literature regarding the influence of emotional states and emotional stimuli on performance of goal-related behaviors and neural activity has yielded discrepant results.

\section{Conflict and Control in Nonemotional Tasks}

One particular function of cognitive control is the selection of a correct response from competing responses. In the color-word Stroop task, subjects are required to name the color in which a color word is displayed (Stroop, 1935). On congruent trials, the color and meaning of the word are the same (e.g., the word blue written in blue). Responses on these trials are generally quick and accurate. On incongruent trials, the color and the meaning of the word are not the same (e.g., the word blue written in red). Performance is slower and less accurate on incongruent trials because of the presence of conflict. During these types of trials, cognitive control is needed for the subject to respond with the correct color-naming response ("red") as opposed to the incorrect and conflicting automatic word-reading response ("blue").
Behavioral studies have shown that responses to incongruent trials preceded by another incongruent trial (iI trials) are considerably faster and more accurate than responses to incongruent trials preceded by a congruent trial (cI trials; Gratton, Coles, \& Donchin, 1992). Performance on iI trials is improved because of a strengthening of selection for action or control. On cI trials, performance is poor, because control is not yet implemented and levels of conflict are high.

In an event-related fMRI study of the color-word Stroop task, Kerns et al. (2004) found that a fast iI trial was associated with high dorsolateral prefrontal cortex (DLPFC) activity and high dorsol anterior cingulate cortex (dACC) activity on the preceding incongruent (cI) trial. This study helped establish the role of the dACC as the conflict monitor and the role of a separate and distinct area, the DLPFC, in subsequent cognitive control. These results have since been replicated in several neuroimaging studies (Egner, Etkin, Gale, \& Hirsch, 2008; Egner \& Hirsch, 2005; Kerns, 2006), and Egner and Hirsch (2005) later showed that DLPFC improves task performance by enhancing processing of the task-relevant stimulus dimension.

\section{Conflict and Control in Emotional Tasks}

The results described above account for the cognitive and neural underpinnings of conflict detection and subsequent control-related adjustments during cognitive tasks. However, in real-world situations, we must con- 
stantly think, react, make decisions, and perform goalrelated behaviors in the presence of emotional stimuli. To best understand how we function in natural situations, it is important to have an understanding of how cognitive and emotional processing interact. Because the cognitive processes underlying the behavioral effects of the Stroop task are well characterized, many researchers have investigated the behavioral and neural mechanisms underlying performance during emotional interference and emotional Stroop tasks as a means for understanding how emotion processing affects cognitive control.

Bishop, Duncan, Brett, and Lawrence (2004) had subjects judge whether two houses were the same or different, while ignoring simultaneously presented task-irrelevant faces that could be either neutral or fearful in emotion. They found rostral ACC (rACC) activity on trials with fearful face distractors that was greater in blocks in which fearful face distractors appeared infrequently. These results parallel those of Carter et al. (2000); rACC activity in Bishop et al. (2004) and dACC activity in Carter et al. appeared strongest in blocks in which conflicting stimuli were least expected and control was least likely to be engaged, suggesting that, like the dACC, the rACC is involved in conflict detection, particularly when conflict (in this case, processing conflict) arises from emotional stimuli.

Etkin, Egner, Peraza, Kandel, and Hirsch (2006) used an emotional facial Stroop task that allowed for trial-totrial analyses comparable to those in Kerns et al. (2004). In their task, subjects were required to respond whether a face was happy or fearful, while ignoring the words happy or fear superimposed over the face. High-conflict (cI) trials activated the amygdala, the caudal ACC, and the DLPFC. Activity in these three regions was predictive of rACC activity on the subsequent trial. rACC recruitment was associated with a reduction in amygdala activity and enhanced task performance on high control (iI) trials. They concluded that amygdala activity is heightened when two conflicting emotional states are represented in the same trial and that the rACC is involved in resolving this conflict via control over the amygdala. Thus, the amygdala and the rACC have separate and distinct roles in conflict detection and control, respectively, during an emotional facial Stroop task, and, unlike Bishop et al. (2004), Etkin et al. (2006) found that the rACC was involved in control over emotional conflict and not in emotional conflict detection.

In a follow-up study, Egner et al. (2008) directly compared conflict- and control-related activity in the emotional facial Stroop task with a similar but nonemotional version of the task. Their emotional task was the same as that used in Etkin et al. (2006), whereas in their nonemotional task, subjects judged whether happy and fearful faces were male or female while ignoring the words male and female superimposed over the face. The two tasks elicited comparable behavioral conflict adaptation effects. As in Egner et al. (2008), high-control trials in the emotional task were associated with activity in $\mathrm{rACC}$, and connectivity analyses showed that rACC activity was associated with decreased amygdala activity. In the nonemo- tional task, high-control trials activated the right lateral prefrontal cortex (LPFC). However, Etkin et al. (2006) found that the amygdala was not significantly more active during high-conflict trials in the emotional task than in the nonemotional task. They also observed overlapping regions of the dACC in response to high-conflict trials in both the emotional and the nonemotional task. These results suggest that although different prefrontal areas are needed to resolve conflict in emotional and nonemotional tasks, the dACC detects conflict regardless of whether the conflict arises from emotional or nonemotional stimuli.

Finally, Ochsner, Hughes, Robertson, Cooper, and Gabrieli (2009) compared behavioral and fMRI results during performance of emotional and nonemotional versions of a verbal flanker task. They found that incongruent (I) trials in comparison with congruent (C) trials activated dACC and DLPFC in both tasks. However, in the emotional task only, the I-C contrast activated rACC. Activity in the $\mathrm{rACC}$ for the $\mathrm{I}-\mathrm{C}$ contrast was positively correlated with behavioral interference effects in reaction time (RT). Because the design did not allow for analyses of trial-to-trial effects as in Etkin et al. (2006) and Egner et al. (2008), it was difficult to conclude whether the rACC was involved in conflict detection or control in this experiment.

From the above summary, it is evident that the results of these studies are not entirely in agreement. Etkin et al. (2006) provide a model of the amygdala as the detector of emotional conflict, whereas the results of Egner et al. (2008) do not provide strong evidence that the amygdala is specifically or preferentially responsive to emotional conflict. Instead, Egner et al. suggested that the dACC is the conflict detector, regardless of the presence of emotion. Both studies, however, did suggest that the rACC is involved in control during emotional Stroop tasks through inhibition of amygdala activity. On the other hand, Bishop et al. (2004) provided evidence that the rACC is involved in emotional conflict as opposed to implementing control over emotional processing. Finally, Ochsner et al. (2009) showed that a comparable dACC/DLPFC network is involved in emotional and nonemotional conflict, with additional recruitment of the rACC (for either conflict or control) when emotional stimuli are present.

Note that although Bishop et al. (2004), Egner et al. (2008), and Etkin et al. (2006) predicted different roles for the rACC - in emotional conflict detection and control- these studies are not directly comparable. In Bishop et al. (2004) the emotional distractors (fearful faces) did not prompt an incorrect response. Thus, emotional conflict was not response conflict, but conflict in the sense that emotional distractors divert processing resources away from the task at hand. In Egner et al. and Etkin et al. (2006), the distracting words had emotional content and produced a competing response. Emotional conflict in these two studies included both response conflict and additional processing conflict due to the emotional attention-grabbing nature of the distracting words. Finally, although Egner et al. and Etkin et al. (2006) were able to relate rACC activity to changes in behavioral performance typical of enhanced control, Bishop et al. (2004) did not 
show lengthened RTs on infrequent fearful face distractor trials to coincide with the increase in activity in the rACC, which would have strengthened their conclusions that the rACC acts as a detector of emotional conflict.

\section{Goal 1: Compare Conflict Detection and Subsequent Control in Nonemotional Versus Emotional Facial Stroop Tasks}

Our first goal in the present experiment is to compare neural activity during performance of a nonemotional with that during performance of an emotional facial Stroop task (see Figures 1A and 1B) to determine whether conflict detection and cognitive control recruit a similar (Egner et al., 2008; Ochsner et al., 2009) or different (Egner et al., 2008; Etkin et al., 2006) network of brain regions. We predict that, because of the presence of response conflict in both versions of our task, the dACC/DLPFC conflict and cognitive control networks will be engaged. We will look at trial-to-trial effects (cI vs. iI) to investigate whether rACC activity, if found in the emotional task, is involved in conflict detection or in control. Incongruent trials in our emotional task, as in Egner et al. and Etkin et al. (2006), also included both response conflict and processing conflict. If distracting emotional words are processed more deeply, because of their emotional content, this should lead to greater response conflict, because increased processing of the emotional word should increase response preparation. Thus, if the $\mathrm{rACC}$ is involved in detecting either type of conflict (or both) produced by emotional distractors, the rACC should show activation on cI trials but not on iI trials. If the rACC is involved in the resolution of either type of conflict (or both), the rACC should be activated on iI trials and not on cI trials.

\section{Trait Anxiety: Effects on}

\section{Emotion Processing and Control}

One way to elucidate how emotional information interferes with goal-oriented behaviors would be to look at a subject population more susceptible to the effects of emotional stimuli. Recent studies have shown that normal subjects high in measures of anxiety may show impairments in behavioral performance, both on emotional tasks (Dresler, Mériau, Heekeren, \& van der Meer, 2009; Fox, 1993; Ladouceur et al., 2009; Lee, Lim, Lee, Kim, \& Choi, 2009; MacLeod \& Rutherford, 1992; Maxwell, Shackman, \& Davidson, 2005; Verkuil, Brosschot, Putman, \& Thayer, 2009) and on nonemotional tasks (Bishop, 2009; Fox, 1993).

Anxiety is associated with enhanced attention to and processing of threatening stimuli (Bar-Haim, Lamy, Pergamin, Bakermans-Kranenburg, \& van IJzendoorn, 2007; Derryberry \& Reed, 2002; MacLeod, Mathews, \& Tata, 1986; Verkuil et al., 2009). For example, MacLeod et al. found that subjects diagnosed with generalized anxiety disorder were faster to respond to a dot that occurred in the location of a threatening word, whereas control subjects were faster to detect a dot appearing in the location previously occupied by a neutral word. Later studies have suggested that these behavioral effects are due not to enhanced vigi- lance, but rather to an inability to disengage from threatening stimuli (Koster, Crombez, Verschuere, \& De Houwer, 2004; Salemink, van den Hout, \& Kindt, 2007).

Neuroimaging studies show that anxiety is associated with greater activation in emotion-processing regions, particularly the amygdala, in response to threatening stimuli (Bishop, Jenkins, \& Lawrence, 2007; Dickie \& Armony, 2008; Etkin et al., 2004; Etkin \& Wager, 2007; Mathews, Yiend, \& Lawrence, 2004). Although enhanced processing of or interference from threat-related stimuli may be automatic, these effects can be reduced through effortful top-down control processes (Derryberry \& Reed, 2002; Mathews et al., 2004; Reinholdt-Dunne, Mogg, \& Bradley, 2009). However, anxiety may be associated with less activation in the rACC during emotional tasks (Bishop et al., 2004; Etkin \& Wager, 2007; Mathews et al., 2004; Shin et al., 2001).

\section{Goal 2: Determine How Trait Anxiety Affects Behavioral Performance and Neural Activity in An Emotional Facial Stroop Task}

For the emotional Stroop task used in the present study, subjects were required to judge whether faces were neutral or fearful in expression while ignoring response congruent or response incongruent words (neutral and fearful, respectively; Figure 1B). We capitalized on individual differences in trait anxiety to show how enhanced attention to emotional stimuli in high-anxiety (HA) subjects can disrupt both performance on the emotional task and cognitive control networks.

In our task, the word appeared slightly prior to the taskrelevant face (see the Method section), thus automatically capturing attention. If HA is associated with impairment in disengaging from a threatening stimulus, we would expect that HA subjects would be impaired specifically on incongruent trials with a neutral face and fearful word, particularly when control is low, as would occur on a cI neutral-face fearful-word trial (Figure 2). Processing of both the neutral face, which prompts the correct response, and the fearful word, which prompts the incorrect response, would result in lengthened RTs and increased dACC conflict-related activity, which could then recruit PFC-mediated cognitive control. If the PFC-mediated control is adequately implemented in the presence of emotional stimuli, responses on subsequent incongruent (iI) trials would be faster and more accurate. However, if the dACC cannot adequately recruit control areas or if control is disrupted by continuing emotional processing, there would not be advantageous adjustments in performance on subsequent incongruent trials. HA subjects would not be impaired on cI fearful-face neutral-word trials, because they would be able to properly disengage from the nonthreatening word neutral to adequately evaluate a threatening fearful face.

\section{Summary of Goals and Hypotheses}

In summary, in the present study, we compared behavior and neural activity during emotional and nonemotional versions of a facial Stroop task. The first goal was to determine whether comparable conflict and 
A

Nonemotional Task

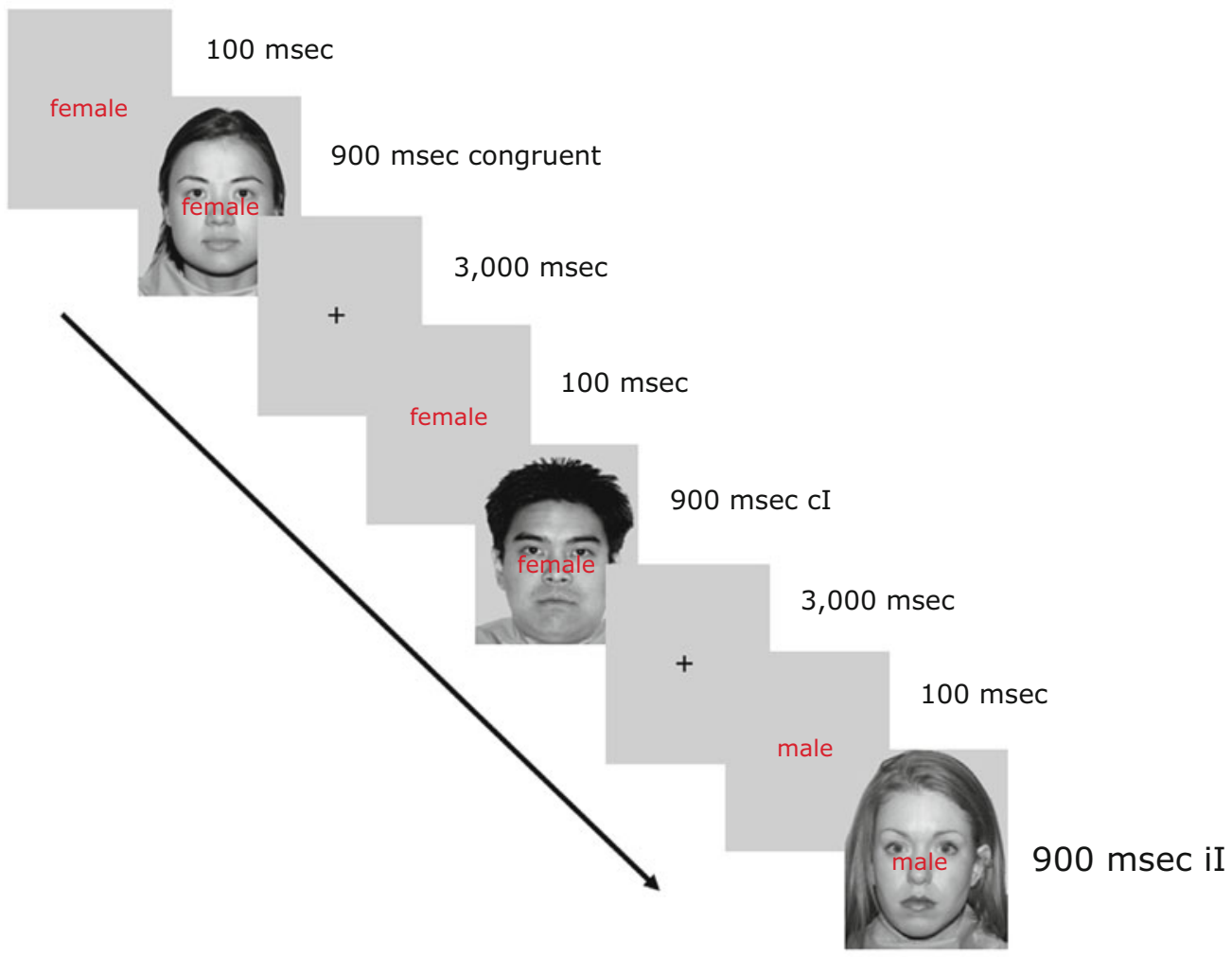

B

Emotional Task

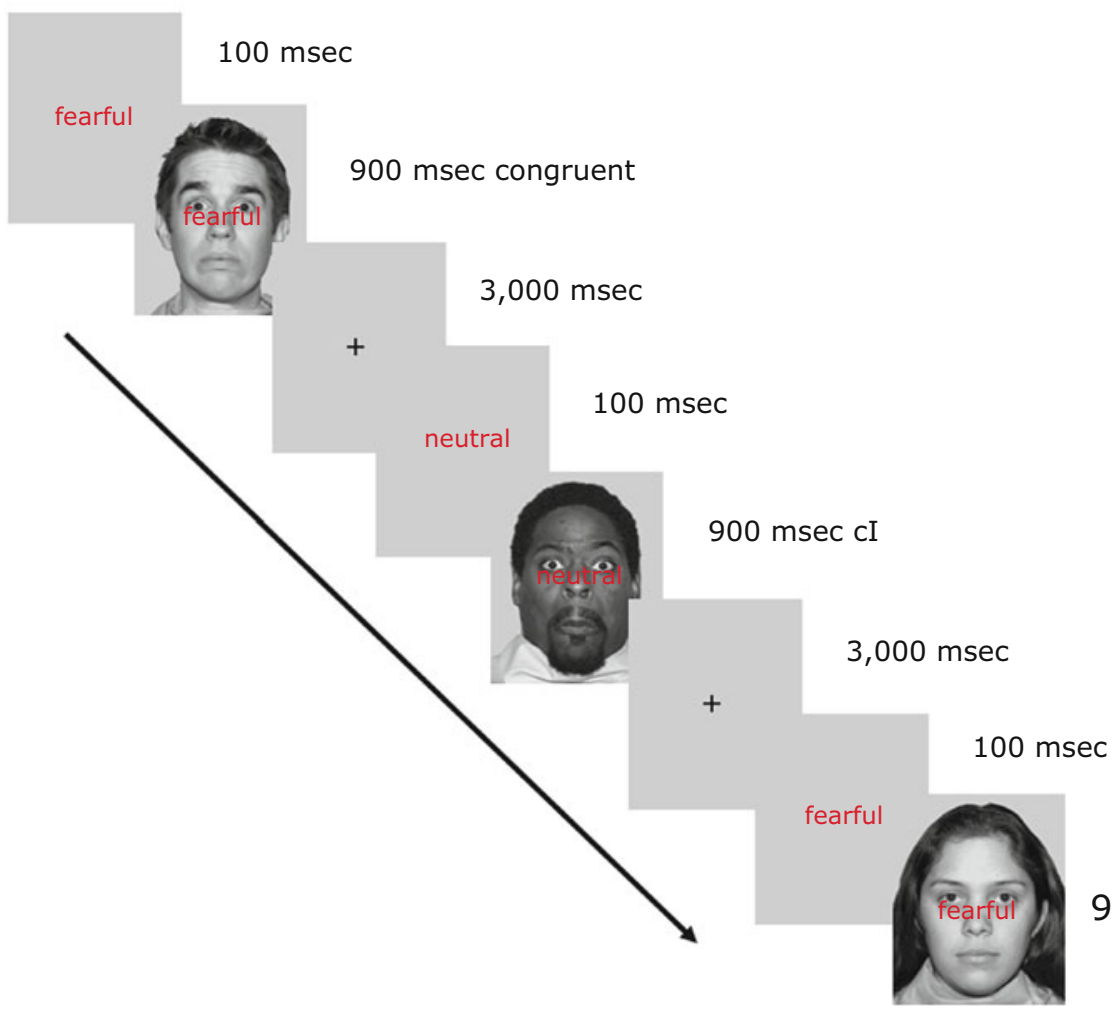

$900 \mathrm{msec}$ iI

Figure 1. Facial Stroop task performed during fMRI data collection. For the nonemotional task, subjects responded whether a face was male or female (A). For the emotional task subjects responded whether a face was neutral or fearful (B). cI, incongruent trial preceded by congruent trial; iI, incongruent trial preceded by incongruent trial. 

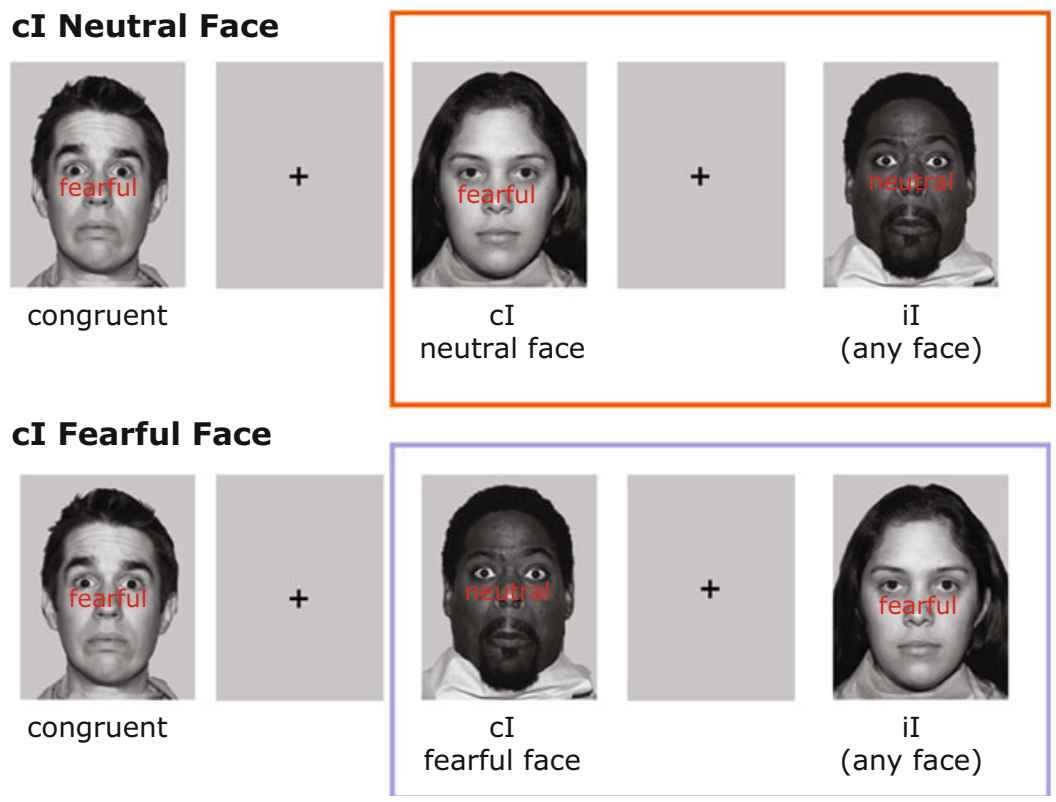

Figure 2. Trial-to-trial congruency effects were investigated by dividing up all incongruent trials on the basis of the congruency of the preceding trial. Incongruent trials preceded by a congruent trial are called $c I$ trials, whereas incongruent trials preceded by incongruent trials are called $i I$ trials. Furthermore, cI trials were divided up on the basis of the valence of the face. iI trials were classified on the basis of whether the previous incongruent (cI) trial was a fearful-face or neutral-face trial. This resulted in four types of trials: cI fearful face, cI neutral face, iI following a cI fearful face, and iI following a cI neutral face.

cognitive control regions are recruited during emotional and nonemotional tasks with the hope of reconciling the disparate findings detailed previously. We predict that a general purpose dACC/DLPFC conflict and cognitive control network is activated during incongruent trials in both tasks, with the possibility that the rACC comes online to implement control over amygdala activity in the emotional task, either in place of or in concert with the DLPFC. Our second goal was to expand on previous studies by determining how trait anxiety affects behavioral performance and neural activity, particularly during the emotional Stroop task. This will help elucidate the neural mechanism of disruption of cognitive control in the presence of an emotional stimulus. We predict that subjects high in trait anxiety will show behavioral impairments, particularly when the fearful stimulus occurs in the task-irrelevant dimension during situations of low control (cI neutral-face fearful-word trials). This may be associated with enhanced activity in emotion-processing areas such as the amygdala and a modulation of dACCmediated conflict detection and/or lateral-PFC-mediated implementation of cognitive control, as well as rACCmediated inhibition of the amygdala.

\section{METHOD}

\section{Subjects}

Potential subjects were recruited from the local university population and the normal control subject pool at the University of California, Davis, Imaging Research Center. All of the subjects were screened prior to the testing session and were invited to participate in the study only if they were right-handed, had no psychiatric or neurological history, were not taking psychoactive medications, had no MRI contraindications, and were native English speakers. Thirtyfour subjects were scanned, of which 4 were excluded. Three were removed because they dosed off or fell asleep in during the task and thus failed to respond for many consecutive trials. One subject was removed because of poor behavioral performance caused by eye fatigue and lack of concentration during the task. For all subsequent analyses, $n=30$ (15 female, 15 male), $M_{\text {age }}=25.4$ years $(S D=$ 4.7 ), range $=20-37$ years. The subjects were paid $\$ 25 / \mathrm{h}$ for the length of the entire testing session.

\section{Materials and Procedure}

Facial Stroop task. Two versions of a facial Stroop task were created using words and face stimuli obtained from the NimStim set (available at www.macbrain.org ${ }^{1}$ ). The words were presented in red 32-point Arial font. All of the face stimuli were converted to grayscale using Adobe photoshop software (Adobe Photoshop CS2 Version 9.0.2)

The subjects performed four blocks of 200 trials: two blocks of a nonemotional task and two blocks of an emotional task (Figure 1). Each trial began with the appearance of a red word, which was presented alone for a brief duration $(100 \mathrm{msec})$ and then followed by a $900-\mathrm{msec}$ presentation of the word and a face together. The word appeared over the center of the face. Trials were separated by $3,000 \mathrm{msec}$ of fixation.

For the nonemotional task, each trial consisted of a male or female neutral face presented along with the word male or female, which could be either response congruent (e.g., a male face with the word male) or response incongruent (e.g., a male face with the word female). For the emotional task, each trial consisted of a neutral or fearful face, along with a response-congruent or responseincongruent word (neutral or fearful). 
For the nonemotional task, the face stimuli consisted of neutral closed-mouth faces from 10 male and 10 female individuals. For the emotional task, the face stimuli consisted of neutral and fearful closed-mouth faces from 5 female and 5 male individuals. The faces used in the nonemotional task were not used in the emotional task (and vice versa). In the nonemotional blocks, each individual's face appeared 10 times (always with a neutral expression), whereas in the emotional blocks, each individual's face appeared 20 times (10 times with a neutral expression and 10 times with a fearful expression). Consequently, repetition of facial stimuli was equivalent in the two tasks, with 20 unique facial stimuli per task.

The subjects received a fixed order of trials for each block. Each block contained $140(70 \%)$ congruent trials and $60(30 \%)$ incongruent trials. Twenty-five of the incongruent trials were preceded by another incongruent trial (iI), whereas the remaining 35 incongruent trials were preceded by a congruent trial (cI) (see Figure 1). Of the 25 iI trials per block, 17 were preceded by only 1 incongruent trial, 7 were preceded by 2 incongruent trials, and 1 incongruent trial was preceded by 3 incongruent trials. In all cases, an incongruent trial preceded by an incongruent trial is referred to as an iI trial, regardless of whether it was preceded by one or more consecutive incongruent trials.

Anxiety assessment. All of the subjects completed the trait portion of the State-Trait Anxiety Inventory (STAI) immediately following completion of scanning (Spielberger, Gorsuch, \& Lushene, $1970)$. The mean trait anxiety score was $31.7(S D=6.6)$, with a range of 20-53. The subjects were split into HA and low-anxiety (LA) groups based on trait anxiety score. For the HA group $(n=$ $15)$, the mean trait anxiety score was $36.5(S D=5.7)$, whereas for the LA group $(n=15)$, the mean trait anxiety score was $25.7(S D=$ 4.0 ), and the trait anxiety score was significantly higher in the HA group than in the LA group $[t(28)=6.006, p<.001]$. The LA group was made up of 9 male and 6 female subjects, whereas the HA group consisted of 9 female and 6 male subjects. A chi-square test determined that this 9:6 gender ratio was not significantly different from an even gender balance $\left[\chi^{2}(1)=.600, p=.439\right]$ and that a 9:6 gender ratio was not significantly different from a 6:9 gender ratio $\left[\chi^{2}(1)=2.5000, p=.114\right]$.

Scanning procedure. Task instructions were administered verbally to the subjects by the experimenter prior to the subjects' entering the scanner. Each subject performed four blocks, which always alternated between the two tasks, and order (nonemotionalemotional-nonemotional-emotional or emotional-nonemotionalemotional-nonemotional) was counterbalanced across subjects. For the nonemotional task, the subjects were to judge whether the faces were male or female, whereas for the emotional task, they were to judge whether the faces were neutral or fearful. They were instructed to ignore the word stimulus. The experimenter informed the subjects that instruction screens prior to each block would indicate whether the imminent block would be the nonemotional task or the emotional task and which finger response button (right index finger or right middle finger) would be used for each response. Fingerresponse mappings were counterbalanced across subjects for each of the two tasks. The subjects were instructed to respond as quickly as possible while maintaining reasonable accuracy, and they were strongly encouraged to emphasize short RTs over highly accurate performance.

The facial Stroop stimuli were presented using Eprime Version 1.2.1.884 (Psychology Software Tools, Pittsburgh, PA; www.pstnet .com) on a standard Dell computer connected to a Sanyo PRO xtraX projector, which displayed the stimulus on a large screen placed at the foot of the scanner bed. The subjects viewed the stimulus via an adjustable mirror attached to the head coil.

Image acquisition. Functional and structural imaging was performed on a 1.5-Tesla magnetic resonance imaging system (Signa Horizon NV/I, OS Version 84M4; GE Medical Systems, Milwaukee, WI). The scanning session included T1-weighted localizer images, four runs of functional imaging, and high-resolution anatomical im- ages. The functional images were collected using a T2*-weighted in-out spiral sequence with a repetition time (TR) of 2,000 $\mathrm{msec}$, an echo time (TE) of $30 \mathrm{msec}$ and a flip angle (FA) of $80^{\circ}$. For each $\mathrm{TR}, 27$ interleaved oblique axial slices were acquired parallel to the AC-PC line (220-mm field of view [FOV], $64 \times 64$ matrix, slice thickness of $5.0 \mathrm{~mm}$ [no gap]). The functional slices did not cover the full brain volume, but slice acquisition was placed such that the rACC regions were acquired and more posterior occipital areas were not covered. The in-out spiral sequence and oblique axial slice acquisition were used to maximize signal-to-noise ratio in the orbitofrontal cortex and the rACC (Glover \& Law, 2001). High-resolution structural images were acquired using a T1-weighted oblique 3D SPGR at a resolution of $.86 \times .86 \times 1.2 \mathrm{~mm}$.

Image preprocessing. The first six images of each block were discarded. The remaining 400 images per block were preprocessed and analyzed using SPM2 software (Functional Imaging Laboratory, University College London, London, United Kingdom, www.fil.ion .ucl.ac.uk/spm). Slice timing correction to the first slice acquired was performed first, followed by realignment of each image to the first image in the series using a rigid-body transformation and sinc interpolation. Next, the high-resolution SPGR was coregistered to the mean functional image using a 6-parameter rigid-body transformation. Functional images were then normalized to a T1 MNI template using the parameters of the coregistered mean functional image, as well as a 12-parameter affine transformation algorithm followed by a nonlinear estimation of deformations. Data were resampled to $3 \times 3 \times 3 \mathrm{~mm}$ voxels. Finally, the functional data were smoothed using an 8-mm FHWM Gaussian kernel. All of the functional data is presented in MNI stereotaxic space.

\section{fMRI Analysis}

The functional data were analyzed in SPM2 using a random effects general linear model (GLM) approach. For each subject, different trial types were entered as covariates and convolved with a canonical hemodynamic response function. Error trials (trials in which an incorrect response was made), posterror trials, and no-response trials (trials in which the subject did not make a buttonpress) were modeled separately but not included in any of the contrasts. Thus, all contrasts included correct trials only.

Conflict and control in nonemotional versus emotional facial Stroop task. Our first GLM was specifically designed to compare and contrast the nonemotional task and the emotional task, with an emphasis on cognitive control-demanding incongruent trials. All correct trials were modeled on the basis of task (nonemotional or emotional) and congruency (congruent [C] or incongruent [I]). Incongruent trials preceded by congruent trials (cI trials) were modeled separately from incongruent trials preceded by another incongruent trial (iI trials).

Beta values for the appropriate trials were used to create contrasts for each individual subject. The individual subject contrasts were then used in the group analysis and were submitted to onesample $t$ tests. We investigated which areas were involved in conflict detection and cognitive control within each of the tasks with the following contrasts: I-C, cI-iI and iI-cI, separately for the nonemotional task and the emotional task. Next, in order to directly compare congruency-related activity between the tasks, we subtracted $\mathrm{I}-\mathrm{C}$ nonemotional task from $\mathrm{I}-\mathrm{C}$ emotional task and vice versa. The contrast (I-C nonemotional task) - (I-C emotional task) was designed to indicate areas with greater activity on incongruent trials in the nonemotional task, while the contrast (I-C emotional task) (I-C nonemotional task) was designed to indicate areas with greater activity on incongruent trials in the emotional task. We did the same for the cI-iI and iI-cI trials to directly compare conflict detection and cognitive control regions between the two tasks, respectively.

Our first GLM was designed to test for differences in cognitive control between the two tasks. In order to avoid false positives and focus on relevant brain regions, we used stringent statistical methods and restricted these analyses to a masked region. A mask was 
created using the WFU PickAtlas (Maldjian, Laurienti, Kraft, \& Burdette, 2003) and included the right and left amygdala, the ACC, cingulate gyrus, and the frontal lobe. The results were thresholded at $p<.005$. AlphaSim (http://afni.nimh.nih.gov/pub/dist/doc/manual/ AlphaSim.pdf) was used to determine a cluster extent threshold to correct for multiple comparisons $(p<.05)$.

Emotional facial Stroop task and trait anxiety. Our second GLM was restricted to the emotional task. We modeled trials on the basis of congruency, congruency of the preceding trial, and face valence (neutral or fearful). We modeled iI trials separately on the basis of whether the preceding cI trial was a cI neutral-face trial or a cI fearful-face trial (Figure 2). Because cI trials are associated with high amounts of conflict and low control, the effects of face type on conflict-related activity were assessed with the contrasts cI fearful face - cI neutral face and cI neutral face - cI fearful face. Because of behavioral differences in conflict adaptation as a function of cI face valence, the following contrasts were run in order to investigate the neural correlates of these behaviors: cI fearful face - iI, and iI - cI fearful face, (the iI trials in these contrasts could be of either face type but had to be following a cI fearful-face trial) and cI neutral face - iI and iI - cI neutral face (once again, the iI trials in these contrasts could be of either face type but had to be following a cI neutral-face trial). These contrasts were designed to help determine which brain regions are involved in conflict detection (cI-iI contrasts) and control (iI-cI contrasts) when conflict is a result of emotional content in the taskrelevant dimension (cI fearful-face trials) or when conflict is caused by emotional content in the task-irrelevant dimension (cI neutral-face trials). As is reported below, we observed some behavioral differences between our HA and LA groups. In order to investigate the neural underpinnings of these differences in performance due to trait anxiety, our contrasts were submitted to two-sample $t$ tests.

Our second GLM was designed to look at more subtle effects involving trial-to-trial adjustments in conflict processing and cognitive control, as well as individual differences in trait anxiety. For these contrasts, we performed region-of-interest (ROI) analyses. We created anatomical ROIs for the right and left amygdala and functional ROIs for our prefrontal regions. Our dACC ROI was centered at ( -92032 ), which is an average of the two dACC activations found in Egner et al. (2008). We created a rACC ROI, (-2 50 18), a DLPFC ROI (-34 36 32), and a VLPFC ROI (-36 16 -6), based on coordinates from Bishop et al. (2004). These coordinates were chosen because Bishop et al. found that high anxiety was associated with less activity in these medial and lateral prefrontal regions. All ROIs had a diameter of $10 \mathrm{~mm}$. All reported results were initially thresholded at $p<.005$ and then corrected to $p<.05$ FDR, small volume corrected (svc). SVC was performed for each ROI individually.

\section{Behavioral Analysis}

Behavioral analyses were performed on RT and accuracy data using SPSS version 16.0 (SPSS, Chicago, IL; www.spss.com). Unless otherwise stated, significance was defined as $p<.05$, two-tailed.

\section{RESULTS}

\section{Behavioral Results}

\section{Conflict and Control in Nonemotional Versus Emotional Facial Stroop Task}

A task (nonemotional or emotional) $\times$ previous trial congruency $(\mathrm{c}$ or i) $\times$ current trial congruency $(\mathrm{C}$ or I) ANOVA was performed on RT and accuracy data.

Task effects. For RT, a main effect of task was found $[F(1,29)=46.2, p<.001]$, with an overall longer mean RT in the emotional task than in the nonemotional task. There was no task $\times$ current trial or task $\times$ previous trial $\times$ current trial interaction, suggesting that congruency effects and trial-to-trial adjustments occur similarly in the two tasks for RT. There was no main effect of task for accuracy, and no task $\times$ current trial $\times$ previous trial interaction. However, there was a current trial $\times$ task interaction $[F(1,29)=5.1, p=.032]$, driven by a larger congruency effect in the emotional task (Figure 3 ). Means and standard deviations for all trial types are shown in Table S1 in the supplementary materials to this article.

Interference effects. For RT, there was a main effect of current trial $[F(1,29)=191.7, p<.001]$, with responses on congruent trials faster than those on incongruent trials. This current trial congruency effect was significant in both the nonemotional task $[t(29)=13.8, p<.001]$ and the emotional task $[t(29)=12.4, p<.001]$. For accuracy, there was a main effect of current trial $[F(1,29)=$ $37.5, p<.001]$, with responses on congruent trials more accurate than those on incongruent trials. The congruency effect was significant in both the nonemotional task $[t(29)=5.2, p<.001]$ and the emotional task $[t(29)=$ $5.2, p<.001]$.

Trial-to-trial effects. For RT, there was a previous trial $\times$ current trial interaction $[F(1,29)=12.0, p=.002]$. It was driven mainly by iC trials having significantly longer RTs than $\mathrm{cC}$ trials for both the nonemotional task $[t(29)=5.2, p<.001]$ and the emotional task $[t(29)=$ $2.5, p=.019]$. The previous trial $\times$ current trial interaction was significant for accuracy $[F(1,29)=10.230, p=$ $.003]$ and was driven by a trend toward increased accuracy on iI trials than on cI trials in both the nonemotional task $[t(29)=1.4, p<.176]$ and the emotional task $[t(29)=$ $2.0, p<.058]$.

Anxiety effects. The subjects were divided into two groups on the basis of STAI trait score: HA and LA. A task (nonemotional or emotional) $\times$ previous trial congruency (c or i) $\times$ current trial congruency (c or i) $\times$ anxiety (HA or LA) ANOVA was performed on both RT and accuracy data. Anxiety did not interact with task or current trial, and the current trial $\times$ task $\times$ anxiety, current trial $\times$ previous trial $\times$ anxiety, and current trial $\times$ previous trial $\times$ task $\times$ anxiety interactions did not reach significance in RT or accuracy.

\section{Emotional Facial Stroop Task and Trait Anxiety}

Interference effects. For the emotional task, a face valence (fearful or neutral) $\times$ anxiety group (HA or LA) $\times$ congruency (congruent or incongruent) ANOVA was significant for RT $[F(1,28)=4.423, p=.045]$. Follow-up analyses showed no significant face $X$ anxiety interaction for congruent trials for RT $[F(1,28)=0.032, p=.860]$. However, the face $X$ anxiety interaction was significant for incongruent trials for RT $[F(1,28)=5.294, p=.029]$. The HA subjects were slower on neutral-face fearful-word incongruent trials than on fearful-face neutral-word trials, whereas the LA subjects were faster (see Table S2 for the means). In addition, the difference in RTs for incongruent neutral-face trials and incongruent fearful-face trials was positively correlated with STAI trait score $(r=.437, p=$ .016).

Trial-to trial effects. Finally, we investigated whether face type (fearful or neutral) affected trial-to-trial adjust- 


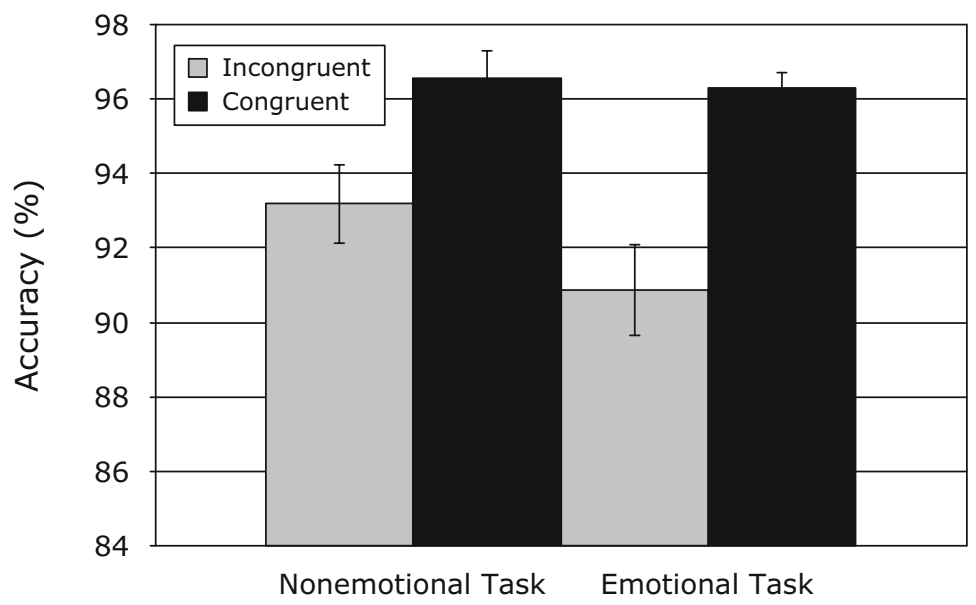

Figure 3. Subjects showed a congruency effect for accuracy in both tasks. There was a larger congruency effect in the emotional task, shown by a significant task $\times$ current trial congruency interaction $[F(1,29)=5.1, p=.032]$. Error bars indicate $\pm 1 S E M$.

ments. In particular, we wanted to see whether face valence on a cI trial affected RT and accuracy on the subsequence incongruent (iI) trial. For this analysis, all incongruent trials were divided up on the basis of the congruency of the preceding trial to form two types of incongruent trials: incongruent trials preceded by a congruent trials (cI) and incongruent trials preceded by incongruent trials (iI). The cI trials were further broken down on the basis of whether they were fearful face (neutral word) or neutral face (fearful word). The iI trials were sorted on the basis of whether the preceding cI trial was a cI fearful-face trial or a cI neutral-face trial. The iI trials could be of either face type (Figure 2).

Face valence affected conflict adaptation differently in the LA and HA subjects. Figure 4A shows RT data for the LA and HA subjects. For the HA subjects, responses on iI trials following a cI neutral-face trial were significantly faster $[t(29)=2.216, p=.044]$, showing evidence of conflict adaptation, whereas responses on iI trials following a cI fearful-face trial were not significantly faster or slower $[t(29)=1.144, p=.272]$. They also showed a nonsignificant trend toward longer RTs for cI neutral-face trials than for cI fearful-face trials $[t(29)=1.786, p=.096]$ and another trend for a cI face type (fearful or neutral) $\times$ trial type (cI or iI) interaction $[F(1,28)=3.425, p=.085]$. For the LA subjects, there were no significant differences among any of the trial types and no interaction between cI face type (fearful or neutral) and cI to iI RT adjustments $[F(1,28)=0.088, p=.771]$.

When the two anxiety groups were directly compared, a trial type (cI fearful face or cI neutral face) $\times$ anxiety group (HA or LA) ANOVA showed a nonsignificant trend $[F(1,28)=2.985, p=.095]$, and the amount of slowing on cI neutral-face trials relative to cI fearful-face trials was positively correlated with STAI score $(r=.355$, $p=.054)^{2}$ (Figure 4B). A trial type (cI neutral face or iI following cI neutral face) $\times$ anxiety group (HA or LA)
ANOVA was significant $[F(1,28)=5.111, p=.032]$, and the amount of iI speeding following a cI neutral-face trial was positively correlated with STAI score $(r=.435, p=$ $.016)^{3}$ (see Figure 4C).

In summary, the HA subjects showed RT slowing on cI neutral-face fearful-word trials. Following these slowed trials, performance was improved, indicated by decreased RTs on iI trials following a cI neutral-face trial. The LA subjects did not show these effects, and correlation analyses indicated that trait anxiety was correlated with these behavioral impairments and subsequent adjustments in cognitive control.

\section{fMRI Results}

\section{Conflict and Control in Nonemotional Versus Emotional Facial Stroop Task}

Interference effects. We subtracted activity during congruent $(\mathrm{C})$ trials from activity during incongruent (I) trials for each of the two tasks. The two tasks activated a qualitatively similar network of lateral and medial frontal cortical regions implicated in cognitive control. For the nonemotional task, four clusters of activity were found, including one large cluster centered in the left precentral gyrus/BA $6(-36963)$ that extended into the dACC and the right lateral PFC, a left lateral PFC cluster (-51 24 0), and a cluster in the posterior cingulate $\left(\begin{array}{lll}-6 & 27 & 30\end{array}\right)$. The emotional task similarly activated a right lateral PFC cluster (54 9 21), a left lateral PFC cluster (-45 18 33), and a dACC cluster (6 18 48) (see Table S3). Figure 5 shows the overlap between the two tasks. Although the extent of activation was greater in the nonemotional task, particularly in the bilateral VLPFC, the tasks activated strikingly similar lateral and medial prefrontal areas. There was no evidence of amygdala or $\mathrm{ACC}$ activation in the emotional task. These results suggest that the two tasks activated comparable DLPFC and dACC networks when cognitive control was required. 


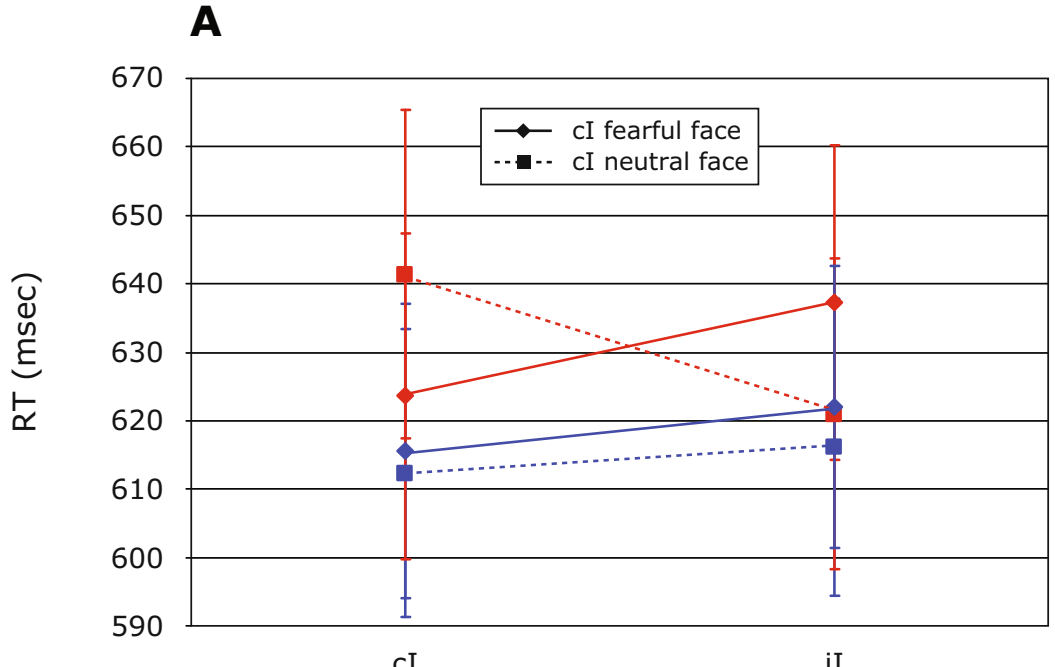

Trial Type

Figure 4A. Face valence affects trial-to-trial adjustments differently for highanxiety (HA; lighter/red) and low-anxiety (LA; darker/blue) groups. The mean response times (RTs) for cI fearful-face trials, iI trials following cI fearful-face trials, cI neutral-face trials, and iI trials following cI neutral-face trials are shown for LA and HA groups (A).

Next we directly compared incongruent trial activity between the two tasks. For the contrast (I-C nonemotional task $)-(\mathrm{I}-\mathrm{C}$ emotional task) there was no significant activity, indicating that no areas showed more activity to incongruent trials in the nonemotional task than in the emotional task. Thus, the larger extent of activity shown in the I-C nonemotional contrast does not represent significantly greater recruitment of regions similar to or different from that in the emotional task. Similarly, for the contrast (I-C emotional task) - (I-C nonemotional task), there were no significant clusters of activation.

Trial-to-trial effects. Contrasts for trial-to-trial adjustments did not reveal much activity. For the nonemotional task, the cI-iI contrast resulted in one cluster of activity in the medial frontal gyrus/BA 6, whereas the iI-cI contrast activated the VLPFC/BA 47 (Table S3). There were no significant clusters for either the cI-iI or the iI-cI contrast for the emotional task. When the cI-iI and iI-cI contrasts were directly compared between tasks (as described above for the $\mathrm{I}-\mathrm{C}$ contrasts), there were no significant regions of activity, suggesting that once again, the nonemotional and emotional tasks do not activate different regions on trials characterized by either high conflict or high cognitive control.

\section{Emotional Facial Stroop Task and Trait Anxiety}

Trial-to-trial effects. For conflict, trait anxiety affected task performance in a very specific way: The HA subjects were slow on cI neutral-face fearful-word trials and showed a decrease in RT (speeding) for iI trials (regardless of face) following cI neutral-face trials. We ran a two-sample (HA or LA) $t$ test on the cI-neutral-faceiI contrast to see if the two anxiety groups differed in conflict-related activity on the cI neutral-face trial. Activity was significantly greater in the dACC ROI for the HA subjects than for the LA subjects (coordinates -32430 , FDR $<.05$, svc; Table S4). A two-samples $t$ test for the cIfearful-face-iI contrast was not significant for the dACC ROI. These effects correspond nicely to the behavioral results. Although the two groups performed equivalently on cI fearful-face trials, the HA subjects were impaired only on the cI neutral-face trials. They showed more dACC activity on these trials and then subsequently showed a conflict-related adjustment.

Some studies have suggested that the rACC is involved in control over emotional conflict (Egner et al., 2008; Etkin et al., 2006). We did not find evidence of rACC activity in typical control contrasts such as iI-cI fearful face or iI-cI neutral face overall, in a between-groups analysis, or in either of our anxiety groups alone. As was discussed previously, subjects low in anxiety were more likely to show rACC-mediated control. However, in this experiment, the LA subjects did not show cI to iI control-related adjustments behaviorally (Figure 4A). Consequently, we decided to also contrast cI neutral-face and cI fearful-face trials. Because the LA subjects did not show RT slowing on cI neutral-face trials relative to cI fearful-face trials (unlike the HA subjects), a cI-neutral-face-cI-fearful-face contrast could indicate activity associated with control over a task-irrelevant emotional stimulus. Although the betweengroups analysis on the cI-neutral-face-cI-fearful-face contrast did not reveal any significant activity, separate analyses of the two anxiety groups showed the predicted effect. The LA subjects showed a 34-voxel cluster of activation in the rACC ROI (coordinates -3489 , FDR $<.05$, svc; Table S4, Figure S1) for the cI-neutral-face-cI-fearful- 


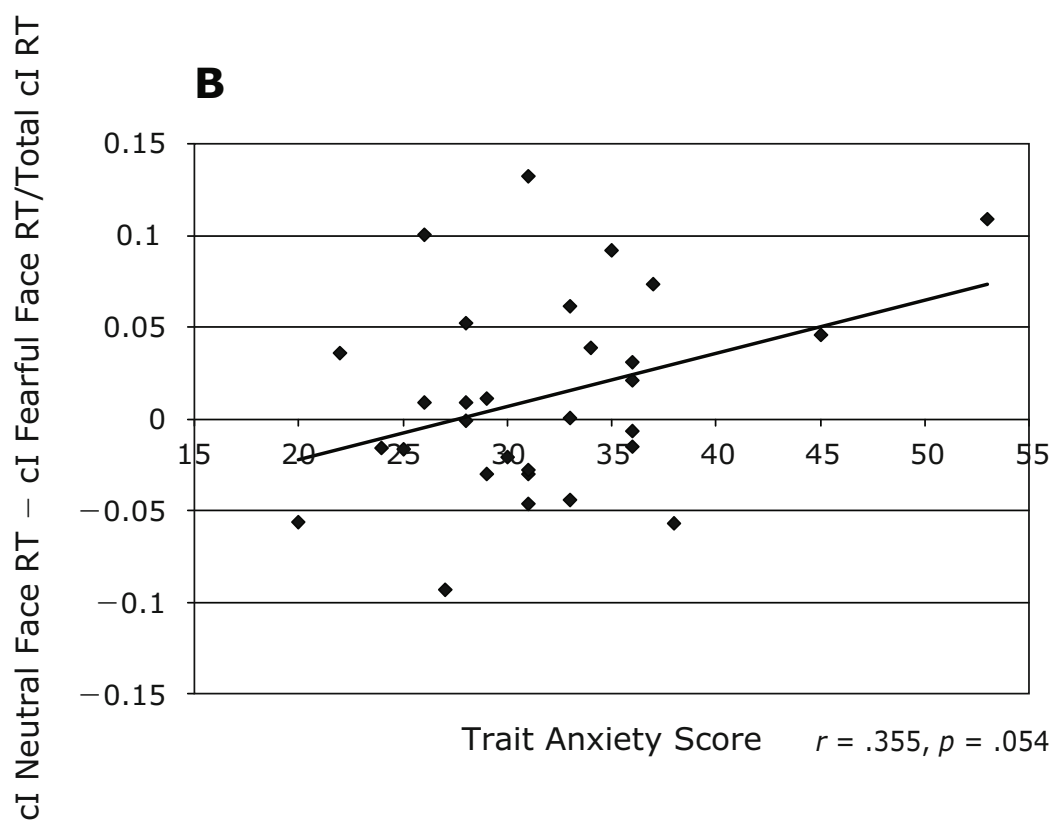

C

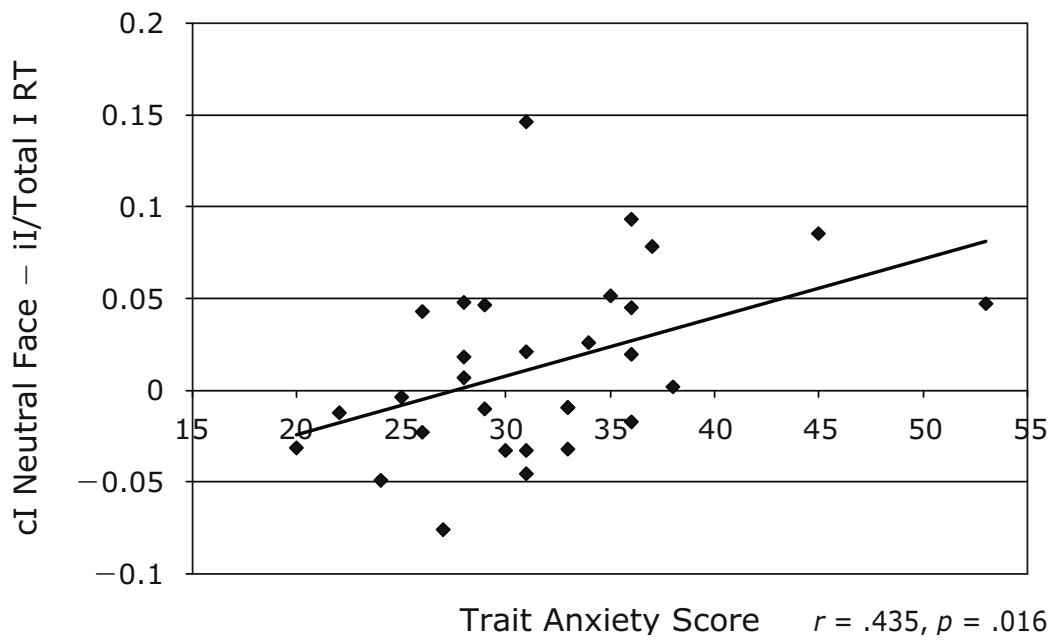

Figures 4B and 4C. For each subject, the mean RT for cI neutral-face trials - the mean RT for cI fearful-face trials was divided by the mean RT for all cI trials. This score was plotted against trait anxiety score (B). The mean RT for cI neutral-face trials - the mean RT for iI trials (following cI neutral-face trials) was divided by the mean RT for incongruent trials. This measure was plotted against trait anxiety score (C). cI, incongruent trial preceded by congruent trial; iI, incongruent trial preceded by incongruent trial; I, incongruent; RT, response time.

face contrast, whereas the HA subjects did not show any activity in the rACC. This suggests that the rACC is selectively involved in the emotional task and that activation of this region reflects individual differences in trait anxiety and behavioral performance associated with control.

Fearful versus neutral faces. Finally, although the behavioral results did not show between-groups differences in response to fearful faces, previous experiments have shown enhanced attention to threat stimuli, which is often mediated by increased amygdala activation (Bishop et al., 2007; Dickie \& Armony, 2008; Etkin et al., 2004;
Etkin \& Wager, 2007; Mathews et al., 2004). We investigated between-groups differences in response to fearful faces during our emotional task. In accordance with this literature, the left amygdala was active more in the HA subjects than the LA subjects for the cI-fearful-facecI-neutral-face contrast (Table S4, Figure 6). In Figure 6, we show the parameter estimates for the significant left amygdala cluster for the cI-fearful-face-cI-neutral-face two-samples $t$ test to illustrate that this interaction was clearly driven by the HA subjects' strong response to cI fearful-face trials. The HA subjects showed more amyg- 


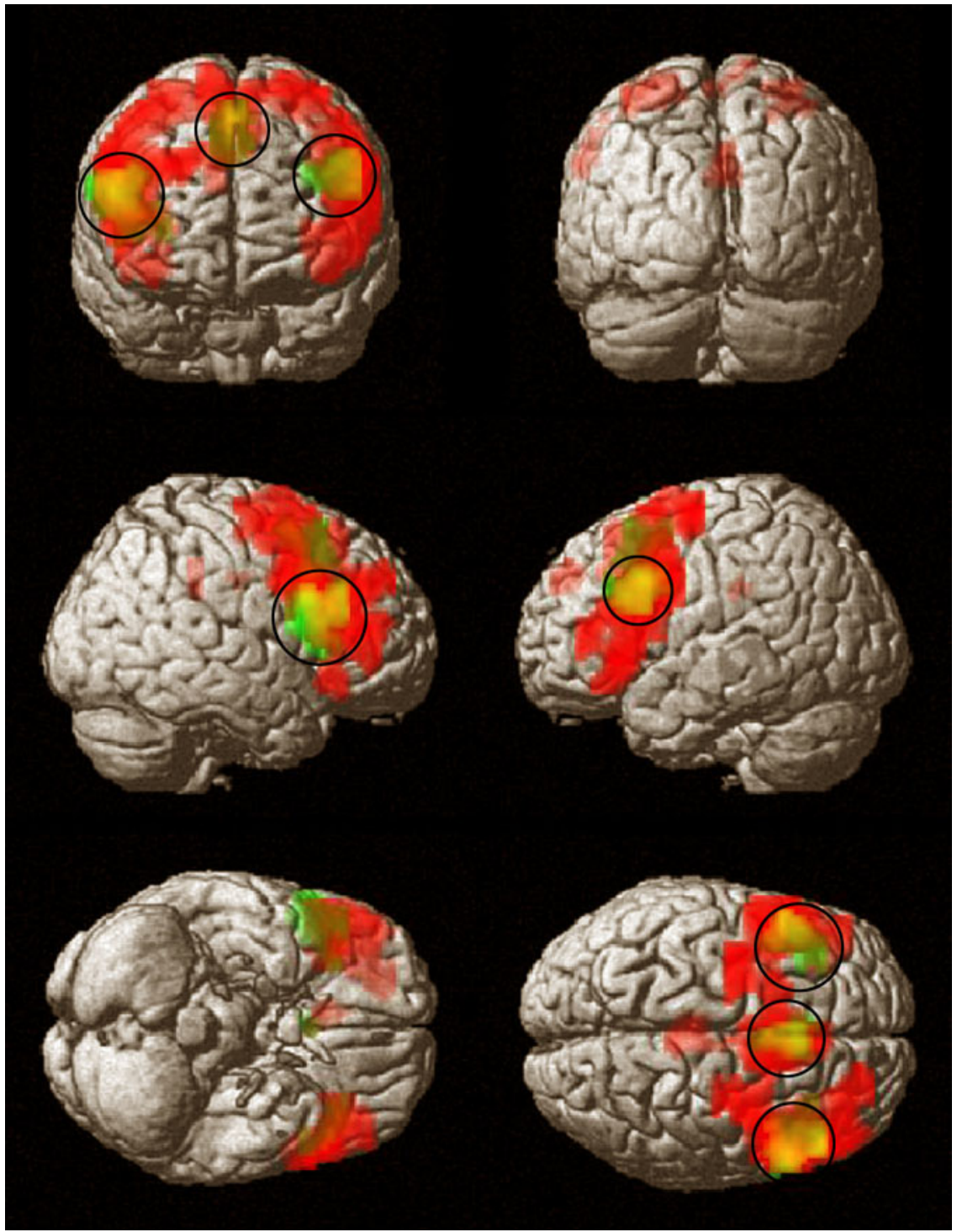

Figure 5. Incongruent trials activate similar prefrontal control areas in the two tasks. Overlap (yellow, circled) between the I-C nonemotional task contrast (red) and the I-C emotional task contrast (green) is shown. Results are displayed at $p<.05$, corrected.

dala activity in response to the cI fearful-face trials than to the cI neutral-face trials $[t(14)=2.721, p=.017]$ and more activity to the cI fearful-face trials than did the LA subjects $[t(28)=2.404, p=.023]$.

\section{DISCUSSION}

\section{Conflict and Control in Nonemotional Versus Emotional Facial Stroop Task}

\section{Behavior}

We compared the behavioral and neural response to emotional and nonemotional facial Stroop tasks in healthy normal subjects. The behavioral results show that the emotional facial Stroop task was more difficult than the nonemotional facial Stroop task, with longer RTs in the emotional task across all trial types and more pronounced congruency interference effects in the accuracy domain. Although it has been reported that nonclinical subject samples are not impaired on emotional Stroop tasks (Whalen et al., 1998; Williams, Mathews, \& MacLeod, 1996), Egner et al. (2008) reported that RTs for their emotional task were significantly longer than those for their nonemotional task and also that the emotional task produced significantly larger trial-to-trial effects. Likewise, our subjects not only showed an overall increase in RTs, but they also showed larger congruency interference effects in the accuracy domain. This result suggests that the effects of emotion on performance are twofold. First, making emotional judgments of faces is overall more difficult than making gender judgments of faces, which result in longer RTs. In everyday life, we frequently evaluate and label a person as belonging to a particular gender. 


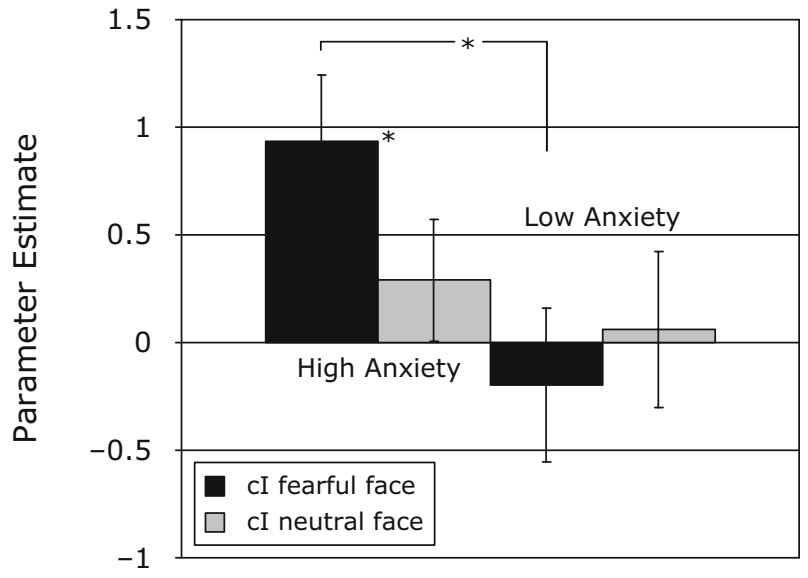

Figure 6. High-anxiety (HA) subjects show enhanced amygdala activity in response to fearful faces. A two-samples $t$ test (HA or low-anxiety [LA]) for the cI-fearful-face-cI-neutral-face contrast revealed activity in the left amygdala $(-21-6-15)$. The mean beta values for this significant cluster are displayed to illustrate what is driving this interaction. cI, incongruent face preceded by congruent face. Error bars indicate \pm 1 SEM. $\quad$ " $p<.05$.

Emotion, however, is more subtle and dynamic, and people are less often given fearful or neutral labels. Second, the presence of a larger congruency interference effect in the emotional task suggests that task-irrelevant words are more likely to elicit incorrect responses when the task is emotional than when it is nonemotional. We conclude that the simultaneous occurrence of emotional content and conflict has an additive effect.

\section{Brain Activity}

Despite our finding that the emotional task was more difficult, we did not find any evidence in our full group data that different conflict and cognitive control regions were recruited during incongruent trials. This was demonstrated in two ways. First, I-C contrasts performed separately for each of the two tasks revealed significant activity in the same regions: the right PFC, the left PFC, and the dACC. Second, I-C contrasts for the two tasks were directly compared and did not reveal any regions that were significantly activated more in one task than in the other. We did not see activation of the rACC in the emotional task in our analysis of the entire subject group. Given that we did see evidence of rACC activation in the LA group, our lack of rACC activation in the full group data could be due to individual differences in rACC recruitment (see below).

One limitation of the present study that warrants discussion is the lack of classic conflict adaptation effects in the behavioral data. Specifically, in both tasks responses on the iI trials were not faster than the those in cI trials. We believe that the lack of trial type effects stems from the instructions used in this task. Instead of being instructed to maintain speed and accuracy during task performance, the subjects were explicitly told to emphasize speed, which has been shown to increase RT interference (I-C) effects (van Veen, Krug, \& Carter, 2008). Between scanning blocks, the subjects were again reminded by the experi- menter to maintain short RTs. This may have prompted a strategy shift toward shorter RTs that masked the more subtle trial-to-trial adjustments in behavior. If subjects are already faster than normal on the high-conflict cI trials, they may not be able to decrease RTs on the iI trial, although accuracy may improve. In fact, for both tasks, responses on the iI trials were more accurate than those on the cI trials. This difference was not significant for the male-female task $[92.7 \%$ vs. $93.9 \% ; t(29)=1.386, p<$ .176] but was almost significant for the neutral-fearful task [90.4\% vs. $91.8 \% ; t(29)=1.975, p<.058]$. In addition, the fact that iI speeding was evident following $\mathrm{cI}$ neutral-face trials in the HA subjects, characterized by lengthened RTs, suggests that these trial-to-trial effects do occur when subjects do not respond quickly.

This strategy adjustment toward speeded responding may also have hindered our ability to detect trial-to-trial adjustments in AACC-mediated conflict detection and DLPFCmediated control in the neuroimaging data. Van Veen et al. (2008) found that a speed-emphasis strategy in the Simon task is characterized by increased sustained baseline levels of dACC and DLPFC activity and reduced transient activation in the dACC and the DLPFC. The increased sustained DLPFC activity in Van Veen et al. showed functional connectivity to motor-preparation areas, suggesting that it enhances response-preparation representations. In our study, these more global strategy-related strategy changes in dACC activity and, most notably, in DLPFC activity may have contributed to a decrease in transient trial-related activity in these regions, making them difficult to detect in our $\mathrm{cI}-\mathrm{iI}$ and iI-cI contrasts.

\section{Emotional Facial Stroop Task and Effects of Trait Anxiety}

\section{Behavior}

In the emotional task, individual differences in trait anxiety clearly influenced behavioral performance in the emotional task in two ways. First, HA was associated with slowing on cI neutral-face fearful-word trials relative to cI-fearful-face neutral-word trials. Second, HA was associated with greater conflict-related adjustments (iI speeding) following a cI neutral-face fearful-word stimulus. The HA subjects may have experienced more conflict from a task-irrelevant fearful-word distractor than did the LA subjects. However, because they did show an advantageous conflict-induced adjustment following the cI neutral-face fearful-word trial, the HA subjects did not appear to be impaired at implementing cognitive control on the following trial.

\section{Brain Activity}

In a two-samples $t$ test, the HA subjects showed more activity in the dACC for cI neutral-face fearful-word trials than did the LA subjects. According to Botvinick, Braver, Barch, Carter, and Cohen's (2001) model, a dACC conflict monitor will show increasing activity when two responses are simultaneously activated at a high level. If responses associated with both the task-relevant face and the task-irrelevant word were activated in the HA subjects, 
this would cause increased dACC activity and lengthened RTs. As was discussed previously, anxious subjects have enhanced attention to threatening stimuli. Specifically, they may have difficulty disengaging attention from a threatening stimulus (Koster et al., 2004; Salemink et al., 2007)in this case, the fear-related word, which in turn may have caused increased response conflict on these trials. The HA subjects seemed to recover easily from the cI neutral-face trial: The following iI trial was significantly faster and comparable to LA performance on this trial type. This suggests that control mechanisms are recruited sufficiently, although the present data do not reveal how this occurs.

High trait anxiety: Greater amygdala activity. The HA subjects showed greater amygdala activity in response to fearful faces than did the LA subjects, suggesting that they engaged in greater emotional processing. The HA subjects did not show significantly greater amygdala activity in response to cI neutral-face fearful-word trials than did the LA subjects. However, a post hoc correlation showed that activity in the left amygdala $(-21-3-24$, 5 voxels) for the cI-neutral-face-iI contrast was positively correlated with the amount of slowing on cI neutral-face trials. In other words, the subjects who are most impaired on the cI neutral-face fearful-word trials show the greatest amygdala activity on these trials. Increased response conflict on cI neutral-face trials could be due to an amygdalamediated enhanced processing of the fearful word.

Low trait anxiety: Evidence for rACC-mediated control of emotion. There is also some evidence that HA subjects lack control processes that LA subjects are able to engage during cI neutral-face trials. In the LA group, the cI neutral-face fearful-word-cI fearful-face neutralword contrast activated the rACC, whereas in the HA group the same contrast did not reveal any such response in the rACC (Figure S1). The LA subjects may be better able to engage rACC-mediated suppression of the processing of fearful words to reduce behavioral interference from the task-irrelevant fearful word. Other studies suggest individual differences in rACC activity. In accordance with our results, behavioral inhibition system (BIS) score was negatively correlated with rACC activity during an emotional encoding task (Mathews et al., 2004), and state anxiety was associated with reduced rACC recruitment during an emotional distractor task (Bishop et al., 2004). Posttraumatic stress disorder (PTSD) is characterized by a lack of activity in the rACC in response to emotional stimuli (Shin et al., 2001). High levels of activity in the rACC can also be predictive of a response to clinical treatment of anxiety disorders and depression (Nitschke et al., 2009; Pizzagalli et al., 2001; Salvadore et al., 2009; Whalen et al., 2008). Greater amounts of rACC activity during emotional tasks may be a resiliency factor, offering protection from later development of clinical anxiety disorders.

\section{Different Types of Conflict}

Note that although we often focus on conflict at the response level, other types of conflict were present in the incongruent trials in our study. Our incongruent stimuli are also incongruent at a semantic level. For example, the word female and a picture of a male face represent two semantically different categories in addition to prompting two different motor responses. In the emotional task, there is semantic and response conflict as well, and the additional attention-grabbing power of the emotional content, which can also be considered a separate and specific type of emotional processing conflict. Our experiment was not designed to distinguish among these different types of conflict. The presence of activity in the dACC and the DLPFC does not necessarily mean that our effects are solely a result of conflict at the response level, as an experiment designed specifically to distinguish between semantic and response conflict showed dACC and DLPFC activation on both response-incongruent trials and semantically incongruent trials (van Veen \& Carter, 2005). Future experiments will need to distinguish between these different levels of conflict, particularly in emotional tasks, to determine how they contribute to the behavioral and neural effects that we have observed.

\section{Trait Anxiety and Control Strategy}

An interesting possible account of the group differences in RTs and dACC activity is that the HA subjects may have a more reactive control strategy, whereas the LA subjects may implement more proactive control (Braver, Gray, \& Burgess, 2007). Braver et al. used their dual mechanisms of control theory to explain variations in cognitive control across time and different tasks, situations, and individuals. A proactive control strategy requires active maintenance of context in preparation for interference or a difficult task trial, whereas a reactive control strategy is defined as less preparative and goal-focused, and recruitment of control occurs precisely at the moment at which it is needed. Braver et al. suggested that dACC activity is characteristic of a reactive control strategy, in that it signals that a situation has just occurred in which there is a need for more PFC-mediated top-down control. dACC activity is not needed during proactive control, because control is sustained and implemented ahead of time (thus, there are not last-minute control emergencies).

Although a proactive strategy is more goal-oriented in terms of optimal task performance, also it is more demanding than a reactive strategy. A reactive strategy, on the other hand, is more optimal for detecting environment changes and alerting attention to distractors. Braver et al. (2007) reviewed evidence that high-BIS-score individuals, who are high in measures of neuroticism and are attuned to punishment and threat cues, may be more likely to adopt a reactive control strategy in favor of enhanced vigilance to potential threats in the environment. Individuals who score high on the behavioral approach system (BAS), on the other hand, are highly attuned to rewards and are more likely to be goal-oriented and to adopt a proactive control approach. During a working memory task, Gray et al. (2005) found that BAS score was negatively correlated with event-related activity in the dACC and the lateral PFC, and Braver et al. cited additional preliminary studies that associated increased dACC activity with BIS 
scores. Fales et al. (2008) found that HA subjects showed more transient and less sustained cognitive control activity during a working memory task, whereas LA subjects showed the opposite effect.

Because the proportion of incongruent trials was low in our task (30\%), by design a reactive control strategy was encouraged. On $70 \%$ of the trials, the word correctly predicted the response, encouraging the subjects to use this word cue to facilitate performance. However, our results indicate that the HA subjects may nonetheless have adopted a more reactive strategy than the LA subjects. It would be interesting to see if HA subjects also show increased dACC activity relative to LA subjects in an emotional Stroop task in which incongruent trials are proportionally more frequent - a design that would encourage a more proactive strategy. If reactive control is characteristic of anxiety, differences between HA and LA subjects in both behavioral performance and neural activity may be even more apparent, especially if HA subjects are not likely or able to switch to a more proactive control strategy.

\section{Conclusions and Future Directions}

In conclusion, our results help clarify the behavioral and neural mechanisms of interference resolution during emotional and nonemotional Stroop tasks. We observed that although the same basic conflict and cognitive control regions were recruited during incongruent trials in both tasks, individual differences in trait anxiety provide clues as to how emotional stimuli, particularly in the task-irrelevant dimension, can have neural and behavioral consequences.

Future studies should be focused more specifically on the disruption of cognitive control by emotional stimuli, with both individual differences within normal populations and various clinical populations including anxiety, major depression, bipolar disorder, and PTSD. Note that the HA subjects in this experiment were not a clinical sample. It will be interesting to see if clinically anxious subjects are unable to make an adaptive behavioral adjustment following a cI neutral-face trial and if a failure to make this adjustment is associated with a breakdown in DLPFC- or rACC-mediated control mechanisms.

Future experiments should also branch out to explore emotions besides fear (e.g., anger, disgust, happiness) and whether emotional stimuli have similar effects in tasks probing different cognitive functions and tasks of varying difficulty and strategic requirements.

\section{AUTHOR NOTE}

This research was supported by Grants R01 MH059883 and R24 MH081807 awarded to C.S.C. We thank Carter Wendelken, Paul Deramo, and Dennis Thompson for assistance with data analysis. Correspondence regarding this article should be addressed to C. S. Carter, Imaging Research Center, UC Davis Medical Center, 4701 X Street, Sacramento, CA 95817 (e-mail: cameron.carter@ucdmc.ucdavis.edu).

\section{REFERENCES}

Bar-Haim, Y., Lamy, D., Pergamin, L., Bakermans-Kranenburg, M. J., \& VAN IJZENDOORN, M. H. (2007). Threat-related attentional bias in anxious and nonanxious individuals: A meta-analytic study. Psychological Bulletin, 133, 1-24. doi:10.1037/0033-2909.133.1.1
Bishop, S. J. (2009). Trait anxiety and impoverished prefrontal control of attention. Nature Neuroscience, 12, 92-98. doi:10.1038/nn.2242

Bishop, S. [J.], Duncan, J., Brett, M., \& Lawrence, A. D. (2004). Prefrontal cortical function and anxiety: Controlling attention to threat-related stimuli. Nature Neuroscience, 7, 184-188. doi:10.1038/ nn1173

Bishop, S. J., Jenkins, R., \& Lawrence, A. D. (2007). Neural processing of fearful faces: Effects of anxiety are gated by perceptual capacity limitations. Cerebral Cortex, 17, 1595-1603. doi:10.1093/ cercor/bh1070

Botvinick, M. M., Braver, T. S., Barch, D. M., Carter, C. S., \& Cohen, J. D. (2001). Conflict monitoring and cognitive control. Psychological Review, 108, 624-652. doi:10.1037/0033-295X 108.3.624

Braver, T. S., Gray, J. R., \& Burgess, G. C. (2007). Explaining the many varieties of working memory variation: Dual mechanisms of cognitive control. In A. R. A. Conway, C. Jarrold, M. J. Kane, A. Miyake, \& J. N. Towse (Eds.), Variation in working memory (pp. 76-106). Oxford: Oxford University Press.

Carter, C. S., Macdonald, A. M., Botvinick, M., Ross, L. L., Stenger, V. A., Noll, D., \& Cohen, J. D. (2000). Parsing executive processes: Strategic vs. evaluative functions of the anterior cingulate cortex. Proceedings of the National Academy of Sciences, 97, 19441948.

Derryberry, D., \& Reed, M. A. (2002). Anxiety-related attentional biases and their regulation by attentional control. Journal of Abnormal Psychology, 111, 225-236. doi:10.1037/0021-843X.111.2.225

Dickie, E. W., \& Armony, J. L. (2008). Amygdala responses to unattended fearful faces: Interaction between sex and trait anxiety. Psychiatry Research, 162, 51-57. doi:10.1016/j.pscychresns.2007.08.002

Dresler, T., Mériau, K., Heekeren, H. R., \& van der Meer, E. (2009). Emotional Stroop task: Effect of word arousal and subject anxiety on emotional interference. Psychological Research, 73, 364 371. doi:10.1007/s00426-008-0154-6

Egner, T., EtKin, A., Gale, S., \& Hirsch, J. (2008). Dissociable neural systems resolve conflict from emotional versus nonemotional distractors. Cerebral Cortex, 18, 1475-1484. doi:10.1093/cercor/bhm179

EgNeR, T., \& Hirsch, J. (2005). Cognitive control mechanisms resolve conflict through cortical amplification of task-relevant information. Nature Neuroscience, 8, 1784-1790. doi:10.1038/nn1594

Etkin, A., Egner, T., Peraza, D. M., Kandel, E. R., \& Hirsch, J. (2006). Resolving emotional conflict: A role for the rostral anterior cingulate cortex in modulating activity in the amygdala. Neuron, 51, 871-882. doi:10.1016/j.neuron.2006.07.029

Etkin, A., Klemenhagen, K. C., Dudman, J. T., Rogan, M. T., Hen, R., Kandel, E. R., \& Hirsch, J. (2004). Individual differences in trait anxiety predict the response of the basolateral amygdala to unconsciously processed fearful faces. Neuron, 44, 1043-1055. doi:10.1016/j.neuron.2004.12.006

ETKIN, A., \& WAGER, T. D. (2007). Functional neuroimaging of anxiety: A meta-analysis of emotional processing in PTSD, social anxiety disorder, and specific phobia. American Journal of Psychiatry, 164, 1476-1488. doi:10.1176/appi.ajp.2007.07030504

Fales, C. L., Barch, D. M., Burgess, G. C., Schaefer, A., Mennin, D. S., Gray, J. R., \& Braver, T. S. (2008). Anxiety and cognitive efficiency: Differential modulation of transient and sustained neural activity during a working memory task. Cognitive, Affective, \& Behavioral Neuroscience, 8, 239-253. doi:10.3758/CABN.8.3.239

Fox, E. (1993). Attentional bias in anxiety: Selective or not? Behaviour Research \& Therapy, 31, 487-493. doi:10.1016/0005-7967 (93)90129-I

Glover, G. H., \& LAw, C. S. (2001). Spiral-in/out BOLD fMRI for increased SNR and reduced susceptibility artifacts. Magnetic Resonance in Medicine, 46, 515-522. doi:10.1002/mrm.1222

Gratton, G., Coles, M. G., \& Donchin, E. (1992). Optimizing the use of information: Strategic control of activation of responses. Journal of Experimental Psychology: General, 121, 480-506. doi:10.1037/0096 $-3445.121 .4 .480$

Gray, J. R., Burgess, G. C., Schaefer, A., Yarkoni, T., Larsen, R. J., \& BRAVER, T. S. (2005). Affective personality differences in neural processing efficiency confirmed using fMRI. Cognitive, Affective, \& Behavioral Neuroscience, 5, 182-190. doi:10.3758/CABN.5.2.182 
Kerns, J. G. (2006). Anterior cingulate and prefrontal cortex activity in an fMRI study of trial-to-trial adjustments on the Simon task. NeuroImage, 33, 399-405. doi:10.1016/j.neuroimage.2006.06.012

Kerns, J. G., Cohen, J. D., MacDonald, A. W., III, Cho, R. Y., STENGER, V. A., \& CARTER, C. S. (2004). Anterior cingulate conflict monitoring and adjustments in control. Science, 303, 1023-1026. doi:10.1126/science. 1089910

Koster, E. H. W., Crombez, G., Verschuere, B., \& De Houwer, J. (2004). Selective attention to threat in the dot probe paradigm: Differentiating vigilance and difficulty to disengage. Behaviour Research \& Therapy, 42, 1183-1192. doi:10.1016/j.brat.2003.08.001

Ladouceur, C. D., Silk, J. S., Dahl, R. E., Ostapenko, L., Kronhaus, D. M., \& Phillips, M. L. (2009). Fearful faces influence attentional control processes in anxious youth and adults. Emotion, 9, 855-864. doi:10.1037/a0017747

Lee, T. H., Lim, S. L., Lee, K., Kim, H. T., \& Choi, J. S. (2009). Conditioning-induced attentional bias for face stimuli measured with the emotional Stroop task. Emotion, 9, 134-139. doi:10.1037/ a0014590

MacLeod, C., Mathews, A., \& Tata, P. (1986). Attentional bias in emotional disorders. Journal of Abnormal Psychology, 95, 15-20. doi:10.1037/0021-843X.95.1.15

MacLeod, C., \& Rutherford, E. M. (1992). Anxiety and the selective processing of emotional information: Mediating roles of awareness, trait and state variables, and personal relevance of stimulus materials. Behaviour Research \& Therapy, 30, 479-491. doi:10.1016/0005 -7967(92)90032-C

Maldjian, J. A., Laurienti, P. J., Kraft, R. A., \& Burdette, J. H. (2003). An automated method for neuroanatomic and cytoarchitectonic atlas-based interrogation of fmri data sets. NeuroImage, 19, 1233-1239. doi:10.1016/S1053-8119(03)00169-1

Mathews, A., Yiend, J., \& Lawrence, A. D. (2004). Individual differences in the modulation of fear-related brain activation by attentional control. Journal of Cognitive Neuroscience, 16, 1683-1694. doi: $10.1162 / 0898929042947810$

Maxwell, J. S., Shackman, A. J., \& Davidson, R. J. (2005). Unattended facial expressions asymmetrically bias the concurrent processing of nonemotional information. Journal of Cognitive Neuroscience, 17, 1386-1395. doi:10.1162/0898929054985437

Nitschke, J. B., Sarinopoulos, I., Oathes, D. J., Johnstone, T., Whalen, P. J., Davidson, R. J., \& Kalin, N. H. (2009). Anticipatory activation in the amygdala and anterior cingulate in generalized anxiety disorder and prediction of treatment response. American Journal of Psychiatry, 166, 302-310. doi:10.1176/appi.ajp.2008.07101682

Ochsner, K. N., Hughes, B., Robertson, E. R., Cooper, J. C., \& GABRIELI, J. D. (2009). Neural systems supporting the control of affective and cognitive conflicts. Journal of Cognitive Neuroscience, 21, 1842-1855. doi:10.1162/jocn.2009.21129

Pizzagalli, D., Pascual-Marqui, R. D., Nitschke, J. B., Oakes, T. R., Larson, C. L., Abercrombie, H. C., et AL. (2001). Anterior cingulate activity as a predictor of degree of treatment response in major depression: Evidence from brain electrical tomography analysis. American Journal of Psychiatry, 158, 405-415. doi:10.1176/appi .ajp.158.3.405

Reinholdt-Dunne, M. L., MogG, K., \& Bradley, B. P. (2009). Effects of anxiety and attention control on processing pictorial and linguistic emotional information. Behaviour Research \& Therapy, 47, 410-417. doi:10.1016/j.brat.2009.01.012

SaleminK, E., van Den Hout, M. A., \& Kindt, M. (2007). Selective attention and threat: Quick orienting versus slow disengagement and two versions of the dot probe task. Behaviour Research \& Therapy, 45, 607-615. doi:10.1016/j.brat.2006.04.004

Salvadore, G., Cornwell, B. R., Colon-Rosario, V., Coppola, R.,
Grillon, C., Zarate, C. A., JR., \& Manji, H. K. (2009). Increased anterior cingulate cortical activity in response to fearful faces: A neurophysiological biomarker that predicts rapid antidepressant response to ketamine. Biological Psychiatry, 65, 289-295. doi:10.1016/j .biopsych.2008.08.014

Shin, L. M., Whalen, P. J., Pitman, R. K., Bush, G., Macklin, M. L., LASKo, N. B., ET AL. (2001). An fMRI study of anterior cingulate function in posttraumatic stress disorder. Biological Psychiatry, 50, 932-942. doi:10.1016/S0006-3223(01)01215-X

Spielberger, C. D., Gorsuch, R. L., \& Lushene, R. E. (1970). Manual for the State-Trait Anxiety Inventory. Palo Alto, CA: Consulting Psychologists Press.

Stroop, J. R. (1935). Studies of interference in serial verbal reactions. Journal of Experimental Psychology, 18, 643-662. doi:10.1037/ h0054651

van Veen, V., \& CARTer, C. S. (2005). Separating semantic conflict and response conflict in the Stroop task: A functional MRI study. NeuroImage, 27, 497-504. doi:10.1016/j.neuroimage.2005.04.042

van Veen, V., Krug, M. K., \& Carter, C. S. (2008). The neural and computational basis of controlled speed-accuracy tradeoff during task performance. Journal of Cognitive Neuroscience, 20, 1952-1965. doi:10.1162/jocn.2008.20146

Verkuil, B., Brosschot, J. F., Putman, P., \& Thayer, J. F. (2009). Interacting effects of worry and anxiety on attentional disengagement from threat. Behaviour Research \& Therapy, 47, 146-152. doi:10.1016/j.brat.2008.11.003

Whalen, P. J., Bush, G., McNally, R. J., Wilhelm, S., McInerney, S. C., JENIKE, M. A., \& RAUCH, S. L. (1998). The emotional counting Stroop paradigm: A functional magnetic resonance imaging probe of the anterior cingulate affective division. Biological Psychiatry, 44, 1219-1228. doi:10.1016/S0006-3223(98)00251-0

Whalen, P. J., Johnstone, T., Somerville, L. H., Nitschke, J. B., Polis, S., Alexander, A. L., ET AL. (2008). A functional magnetic resonance imaging predictor of treatment response to venlafaxine in generalized anxiety disorder. Biological Psychiatry, 63, 858-863. doi:10.1016/j.biopsych.2007.08.019

Williams, J. M., Mathews, A., \& MacLeod, C. (1996). The emotional stroop task and psychopathology. Psychological Bulletin, 120, 3-24. doi:10.1037/0033-2909.120.1.3

\section{NOTES}

1. Development of the MacBrain Face Stimulus Set was overseen by Nim Tottenham and supported by the John D. and Catherine T. MacArthur Foundation Research Network on Early Experience and Brain Development. Please contact Nim Tottenham at tott0006@tc.umn.edu for more information concerning the stimulus set.

2. Removal of the data point representing the highest trait anxiety score (a possible outlier) rendered the correlation not significant $(r=$ $.201, p=.297)$.

3. When two possible outliers were removed (the data point with the highest trait anxiety score and the data point with the highest RT difference score), the correlation still approached significance $(r=.357$, $p=.062)$.

\section{SUPPLEMENTAL MATERIALS}

Additional behavioral and fMRI results from this study may be downloaded from http://cabn.psychonomic-journals.org/content/ supplemental.

(Manuscript received August 13, 2009; revision accepted for publication March 30, 2010.) 